This article was downloaded by: [Rieh, Soo Young]

On: 5 March 2010

Access details: Access Details: [subscription number 919687867]

Publisher Taylor \& Francis

Informa Ltd Registered in England and Wales Registered Number: 1072954 Registered office: Mortimer House, 3741 Mortimer Street, London W1T 3JH, UK

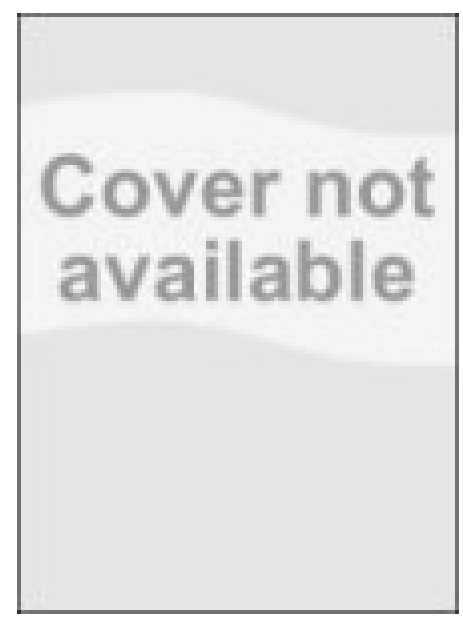

Encyclopedia of Library and Information Sciences, Third Edition

Publication details, including instructions for authors and subscription information:

http://www.informaworld.com/smpp/title $\sim$ content=t917508581

\title{
Credibility and Cognitive Authority of Information
}

Soo Young Rieh ${ }^{\text {a }}$

a School of Information, University of Michigan, Ann Arbor, Michigan, U.S.A.

Online publication date: 09 December 2009

To cite this Article Rieh, Soo Young(2010) 'Credibility and Cognitive Authority of Information', Encyclopedia of Library and Information Sciences, Third Edition, 1: 1, 1337 - 1344

\section{PLEASE SCROLL DOWN FOR ARTICLE}

Full terms and conditions of use: http://www.informaworld.com/terms-and-conditions-of-access.pdf

This article may be used for research, teaching and private study purposes. Any substantial or systematic reproduction, re-distribution, re-selling, loan or sub-licensing, systematic supply or distribution in any form to anyone is expressly forbidden.

The publisher does not give any warranty express or implied or make any representation that the contents will be complete or accurate or up to date. The accuracy of any instructions, formulae and drug doses should be independently verified with primary sources. The publisher shall not be liable for any loss, actions, claims, proceedings, demand or costs or damages whatsoever or howsoever caused arising directly or indirectly in connection with or arising out of the use of this material. 


\title{
Credibility and Cognitive Authority of Information
}

\author{
Soo Young Rieh \\ School of Information, University of Michigan, Ann Arbor, Michigan, U.S.A.
}

\begin{abstract}
This entry defines the concepts of information credibility and cognitive authority, introduces the key terms and dimensions of each, and discusses major theoretical frameworks tested and proposed in library and information science (LIS) and related fields. It also lays out the fundamental notions of credibility and cognitive authority in historical contexts to trace the evolution of the understanding and enhancement of the two concepts. This entry contends that the assessment of information credibility and cognitive authority is a ubiquitous human activity given that people constantly make decisions and selections based on values of information in a variety of information seeking and use contexts. It further contends that information credibility and cognitive authority assessment can be seen as an ongoing and iterative process rather than a discrete information evaluation event. The judgments made in assessment processes are highly subjective given their dependence on individuals' accumulated beliefs, existing knowledge, and prior experiences. The conclusion of this entry suggests the need for more research by emphasizing the contributions that credibility and cognitive authority research can make to the field of LIS.
\end{abstract}

\section{INTRODUCTION}

This entry traces the evolution of the concepts of credibility and cognitive authority in library and information science (LIS) and other related fields including communication, human-computer interaction, and psychology. It provides definitions for information credibility and cognitive authority. It also introduces multiple dimensions as well as theoretical frameworks explaining the process of assessing credibility and cognitive authority, concluding by discussing the significance of the two concepts and calling for further research in the area.

Historically, credibility and cognitive authority of information were considered as criteria for making relevance judgments in LIS. Due to the recent growth of the Internet and concomitant evolution of information, communication, and publishing mechanisms, LIS researchers and practitioners have increasingly recognized the importance of understanding credibility and cognitive authority as a research agenda in its own right. Today people have access to a wider range of information resources than ever before, and as a result, face greater challenges in evaluating the usefulness of information with which they interact. Given the popularity of various self-published resources in which the source of information is vague and uncertain, the assessment of information credibility and cognitive authority has become a ubiquitous human activity.

In this entry, credibility and cognitive authority are considered as closely related yet different concepts. Most definitions of credibility revolve around the concept of believability of information. ${ }^{[1]}$ This entry uses the term cognitive authority, coined by Patrick Wilson to differentiate it from administrative authority. ${ }^{[2]}$ Unlike a person in administrative authority, the world's leading authority in a domain area has no power to command. Experts perceived as not only credible or worthy of belief but also influential in other people's thinking are termed cognitive authorities. Those people or information sources considered to be credible serve as the potential pool of cognitive authorities. That is, cognitive authority is one of the principal aspects of information credibility.

\section{CREDIBILITY DEFINITIONS AND ORIGINS}

\section{Definitions}

Credibility is an intuitive and complex concept. ${ }^{[3]}$ Rather than having one clear definition, credibility has been defined along with dozens of other related concepts such as believability, trustworthiness, fairness, accuracy, trustfulness, factuality, completeness, precision, freedom from bias, objectivity, depth, and informativeness. Most credibility researchers agree that credibility assessment results from simultaneously evaluating multiple dimensions. Among these, two key dimensions are identified: trustworthiness and expertise. ${ }^{[4]}$ Trustworthiness is a core dimension in credibility assessment that captures the perceived goodness and morality of the source. ${ }^{[5]}$ The perception that a source is fair, unbiased, and truthful contributes to the trustworthiness of information. Trustworthiness is, however, not a synonym for credibility because people also must recognize expertise in order to deem information credible. Expertise reflects perceived knowledge, skill, and experience of the 
source ${ }^{[5]}$ Expertise is likewise an important factor given its close relationship to people's perceptions of a source's ability to provide information that is both accurate and valid.

Trustworthiness and expertise are not always perceived together. An expert with the title of doctor or professor might have a reputation of being knowledgeable in a certain area but still might not be considered trustworthy for the tendency to unreliability or bias. A person may think of a friend as being honest and trustworthy in general, but the advice that the friend gives is not necessarily considered credible for the friend's lack of expertise. The most credible information is found in those perceived to have high levels of trustworthiness and expertise.

Credibility does not reside in an information object, source, or person, although the characteristics of the foregoing can serve as the bases for people's assessment. It is people who ultimately make judgments of information credibility. People who have their own experience, knowledge, and beliefs are likely to make their own credibility judgments. Many studies show, in fact, that judgments of credibility are highly subjective assessment processes. In this entry, credibility is defined as people's assessment of whether information is trustworthy based on their own expertise and knowledge.

\section{Historical Development}

The fields of communication and LIS are both concerned with the credibility assessment of information and sources. Credibility research, however, has evolved in fundamentally different ways, with each field having its own origins, approaches, and goals for the study of credibility over the past five decades. Some researchers believe that scholarly interest in credibility dates back to Aristotle's examination of ethos (appeal based on character), pathos (appeal based on emotion), and logos (appeal based on logic or reason). ${ }^{[6]}$ Aristotle's notion of credibility focused mainly on the characteristics of ethos, which is "the communicator's ability to inspire confidence and belief in what was being said" (pp. 422-423). ${ }^{[3]}$

Scholarly examination of credibility began in the twentieth century when psychologists investigated persuasion as a part of the propaganda efforts during the World Wars. A series of landmark studies were conducted by the Yale Group which defined credibility as a receiver-based construct and suggested that credibility is determined by an audience's acceptance of a speaker. ${ }^{[4]}$ In the 1950s, mass media professionals took an interest in the notion of credibility when television became popular and subscription rates for newspapers began to decline. Professional news organizations' examination of perceived credibility of newspapers versus television then grew into the study of media credibility which to this day remains an established research area in the field of communication.

Credibility research began much more recently in LIS as compared to the communication field. This does not mean that LIS researchers and practitioners had no concerns about credibility assessment in the past. Rather, LIS research into human judgments of information centered on the notion of relevance. Relevance is often seen as playing a significant, underlying, and yet elusive role for various information activities such as acquiring, organizing, storing, preserving, searching, communicating, interacting with, and using information. ${ }^{[7]}$ The history of relevance started with the first libraries as library users were concerned about the problem of finding information relevant to them. ${ }^{[7]}$ However, the notion of relevance remained hidden and implicit until Vickery's presentations at the 1958 International Conference on Scientific Information. ${ }^{[8]}$ Since then, relevance has been studied extensively in terms of frameworks for defining relevance dimensions, forms of document representation affecting relevance judgment, subjectivity of relevance judgment, and identification of criteria adopted by users in judging relevance.

While the majority of relevance research has discussed relevance in terms of its topical aspect, which concerns itself with whether or not the topic of a search query matches the topic of a document, alternative notions have been suggested. For instance, utility, which is entirely subjective and based on personal judgment, is proposed as a measure of information retrieval effectiveness. ${ }^{[9]}$ Relevance and utility are not the same concepts. Until the 1980s, relevance was viewed as judgments concerned with aboutness, pertinence, or topical-relatedness. Utility was considered a broad concept involving not only topic-relatedness but also quality, novelty, importance, credibility, and other evaluations. In the 1990s, a substantial body of empirical studies on relevance criteria was conducted, producing studies which consistently revealed that people use much more diverse criteria than mere topicality in making relevance judgments. The criteria identified in these user-centered relevance studies include credibility, authority, completeness, depth, currency, accuracy, quality, effectiveness, belief, and clarity. ${ }^{[10]}$

In LIS, as in other related fields, the emergence and proliferation of information technology, the Internet in particular, provided the impetus for improved and more formal understanding of the notion of credibility. Empirical studies showed that people became more concerned about quality, credibility, and authority of information as they gain awareness of the fact that the Web lacks quality control mechanisms in contrast to traditional information retrieval systems. ${ }^{[11]}$ Further, when looking for information, people rely on multiple information resources and may even seek to verify across different resources given the availability and accessibility of various types of digital media and forms of information. ${ }^{[12]}$ The two fieldsLIS and communication - that have studied credibility from different perspectives and presumptions because of historical origins have drawn closer together than ever before as both fields have paid more attention to the significance of studying credibility and authority assessment in the contemporary digital information landscape. 


\section{TYPOLOGY OF CREDIBILITY}

Communication researchers have traditionally drawn distinctions between source credibility, message credibility, and media credibility. Source credibility usually refers to "judgments made by a perceiver concerning the believability of a communicator" (pp. 130-131). ${ }^{[13]}$ Numerous empirical studies have investigated the dimensions of source credibility from the perspectives of message recipients and identified those factors that might influence audience perceptions of trustworthiness and expertise. These factors include dynamism, composure, sociability, qualification, reliability, animation, poise, and goodnaturedness. ${ }^{[14]}$ A meta-analysis of 114 credibility studies revealed that source expertise is a stronger influence on persuasion than other source characteristics given that the expertise dimension of source credibility is more objective than other dimensions and consequently easier to assess. $^{[15]}$

Message credibility examines how message characteristics such as content, structure, language, and presentation can impact perceptions of the believability of information. But the distinction between message credibility and source credibility is not always clear. Credible sources are likely to produce credible messages, and credible messages are likely to be seen as originating from credible sources. When people have strong interest and involvement, message characteristics become more influential than source characteristics because they are motivated to scrutinize message content. In some situations in which little information is available about the source of a message, people tend to turn to message cues in making credibility judgments. ${ }^{[16]}$ Researchers have examined the influence of various factors such as message comprehensibility, number of arguments, incentives, fear appeals, one-sided versus two-sided messages, repetition, and presentation style on people's message credibility assessments. ${ }^{[14]}$

Media credibility focuses on the relative credibility of various media channels through which a message is sent. Media credibility studies typically ask which medium people would believe if they received conflicting reports on the same news story from different media such as radio, television, magazines, and newspapers. ${ }^{[17]}$ This question aimed to discover people's perceptions of the relative credibility of different news media. Previous studies suggest that media credibility is strongly related to the frequency with which people use a particular medium. In them, people judge their preferred medium as being the most credible.

In recent years, media credibility studies have attempted to compare people's perceptions of traditionally-delivered information sources (newspapers, magazines, brochures, etc.) and their online counterparts (online newspapers, online news magazines, online political Web sites, etc.). The popularity and unique characteristics of the Web as a medium led to studying Web credibility as a distinct notion. Credibility researchers have proceeded to note the following characteristics of the Web: lack of filtering mechanisms, form inclusive of interaction techniques and interface attributes, source ambiguity, and infancy as a medium. ${ }^{[18]}$

Computer credibility impacts the field of humancomputer interaction (HCI) when computers act as knowledge repositories, instruct users, report measures, report on work performance, report on their own state, run simulations, and help in the rendering of virtual environments. Computer credibility assessment relies on simultaneous evaluation of four types of credibility: presumed credibility, reputed credibility, surface credibility, and experienced credibility. ${ }^{[1]}$ Presumed credibility refers to the extent to which people believe information because of general assumptions in their mind. Sometimes people make assumptions based on stereotypes rather than on truth, and these assumptions and stereotypes all contribute to credibility perceptions. Reputed credibility describes the extent to which people believe information because of what third parties-other people, media, or institutions-have reported. These reports may come in the form of endorsements, awards, or referrals. Surface credibility derives from simple inspection. People make credibility judgments based on first impressions of surface traits such as of book covers, the visual designs of software, interface designs, and the information architecture of Web sites. Experienced credibility refers to the extent to which people believe information based on their first-hand experience. It may prove to be the most powerful form of credibility because it derives from people's interaction with others or with systems over an extended period of time.

Some credibility researchers who consider the processes of social endorsement to be crucial in credibility construction have proposed several variants of credibility including conferred credibility, tabulated credibility, and emergent credibility. ${ }^{[6]}$ Conferred credibility indicates that people sometimes recognize credibility not based on a real source of information but on other sources' positive reputation, all of which helps alleviate skepticism. For instance, Google might confer its credibility on sponsored links in the search results page because most people are unaware of the sponsorship model and consider such links equivalent to Google's results. Tabulated credibility refers to the assessments people make based on peer ratings of an individual, organization, or product. The availability of aggregated ratings from other people may widen the range of social input on which people can rely in judging credibility. Emergent credibility arises from a pool of resources, such as Wikipedia, wikis, social networking sites, and other applications created by individuals. These forms of credibility suggest that people are not isolated evaluators of credibility as well as that social engagements and interactions must be considered in understanding credibility construction and assessment. 


\section{AUTHORITY}

Authority has been defined and discussed in many different forms across numerous disciplines, including philosophy, education, psychology, political science, law, religion, and LIS. Authority is related to those areas of competence or applicability over which it is exercised. Having authority is different from being an expert because authority is a relationship involving at least two people. A person can be an expert even though others may not realize or recognize the fact. No individual by himself or herself can be an authority. All authority is "a relation among a bearer, a subject, a field, in virtue of a particular quality, attribute, and context" [19, p. 77]. In other words, authority is limited to spheres. A person can speak with authority within one sphere, but with no authority on questions outside that sphere.

Many researchers agree on the two broad categories of authority: epistemic authority and deontic authority. The former corresponds to being an authority and the latter to being in authority. ${ }^{[19]}$ As an example of epistemic authority, when people say, "He is an authority on Hegel," they mean that he is superior to others within a field in light of knowledge about Hegel. Deontic authority takes the forms of imperial authority as exercised by a state through its government and its various organs, paternalistic authority as exercised by parents over their children, and operative authority as vested in any designated leader or office. In place of epistemic authority and deontic authority, cognitive authority and administrative authority can be used as alternative terms in distinguishing more clearly these two different types of authority. ${ }^{[2]}$

\section{COGNITIVE AUTHORITY}

\section{Definitions}

Cognitive authority is a kind of influence. ${ }^{[2]}$ Those who are cognitive authorities profoundly influence others' thoughts. People who are not cognitive authorities may still exert an influence in the world. What distinguishes people who are cognitive authorities from those who are not is that the formers' influence is recognized in some official manner. Cognitive authority is a matter of degree; thus the weight that a cognitive authority's words carry for others might vary. Even though it is possible for an absolute cognitive authority in a given sphere to have an answer to virtually all of the questions within the area, people in general tend to take the opinions and advice of others with different degrees of seriousness. Cognitive authorities are those people whose opinions and advice are taken more seriously with more weight being placed on their words than on the words of others. ${ }^{[2]}$

Two bases for cognitive authority need to be recognized: being an expert and being reputable. ${ }^{[2]}$ To qualify as an expert, a person should show evidence of knowledge, skill, experience, training, and education. Because knowledge and skill are difficult to test directly, evidence in terms of occupational roles or advanced degrees often must provide support for claims to expert status. However, not all experts necessarily possess outstanding competence. Experience and education may provide evidence of basic qualifications but still be insufficient in providing a high degree of expertise. To be an outstanding expert, a person must have a reputation for that expertise. In addition, established cognitive authorities can transfer authority to other people. When reputation among peers is unknown, special groups of people already considered to be knowledgeable can be turned to. For instance, individual $\mathrm{A}$ believes individual $\mathrm{B}$ because individual $\mathrm{A}$ believes group $\mathrm{C}$, and group $\mathrm{C}$ says that individual $\mathrm{B}$ can be believed. This rule - that one can trust those who are trusted by those one trusts ${ }^{[2]}$ — constitutes a central feature of cognitive authority.

\section{Bases for Assessing Cognitive Authority}

When people acquire influence from cognitive authorities, there should be an answer for the question "What makes you think so?" Whatever the reasons for thinking that certain others deserve cognitive authority, it is not for the reason that people always directly test the authorities' knowledge. Rather, people often cite indirect tests that serve as the bases of cognitive authority judgments. Not only individuals are recognized as cognitive authorities: books, journals, newspapers, manuscripts, and films are all possible sources of knowledge and opinion though they give rise to the same kind of questions about cognitive authority: which works can be taken seriously?; how much weight can be given to what the texts say? When people have sufficient knowledge of certain topics, they can claim directly against what they already know. However, most texts discuss topics people do not know enough about to apply the direct test. This is because people look for information and consult texts to find what they do not know. ${ }^{[20]}$ Therefore, people apply various indirect tests for recognizing the cognitive authority of a text, including personal authority, institutional authority, textual type authority, and intrinsic plausibility. ${ }^{[2]}$

The first apparent basis for recognizing a text's cognitive authority is the cognitive authority of its author. People will trust a text written by an individual or group of individuals whom they trust. The tests of personal cognitive authority are based on present reputation and accomplishments. Recognition of personal authority does not automatically transfer to past or future work. An established reputation is insufficient to establish the current authority of old texts.

Another kind of test is associated with the publisher and publication history. A publishing house can acquire a kind of cognitive authority if it is considered to be good at publishing high-quality work. Thus, publications by a 
publishing house people respect constitute a kind of personal recommendation. A journal can exert authority, too, which transfers to the articles it publishes. Other institutional endorsements are also used as tests of authority. For instance, sponsorship by a learned society or a professional organization serve as the basis of institutional authority, as could publication by a government agency. Then again, published reviews furnish a special indirect test. If a reviewer has cognitive authority then his or her review itself constitutes a personal recommendation.

The third kind of test, that of text type, is based on the text itself separate from its author or publisher. For instance, reference works such as dictionaries and encyclopedias do not draw attention to their compilers as people often do not know who the authors or editors are. Standard reference works tend to be revised frequently and may be considered as institutions in their own right.

Finally, the test of intrinsic plausibility is always available. Reading a few words or sentences of the text may be sufficient for people to decide whether or not to continue reading the entire text. Such rapid assessment is not entirely based on intrinsic plausibility, but does constitute a major part. If people find that a work represents a school of thought they reject or has a style of research they think worthless, they become discouraged from continuing to read the work. Although people do not always reject what they see as being in conflict with their prior beliefs and cognitive positions, they cannot avoid assessing the text's contents as plausible or implausible and accordingly bestowing or withholding cognitive authority.

\section{THE PROCESS OF ASSESSING CREDIBILITY AND COGNITIVE AUTHORITY}

Several theoretical frameworks suggest that the assessment of credibility and cognitive authority needs to be understood as a process rather than a discrete evaluative event. Most of these frameworks and models are developed in the context of Internet use and Web searching. Rieh's Model of Judgment of Information Quality and Cognitive Authority was proposed based on an empirical study conducted in the context of Web searching. ${ }^{[11]} \mathrm{Her}$ model captures the point at which people make predictive judgments about which Web site contains credible information and then follows through to include evaluative judgments by which they express preferences for information encountered. When people open a Web browser, they take the first action based on a predictive judgment that must be made before a new page is introduced. The selection of a certain Web site is based on people's knowledge and experience, recommendations from others, or other characteristics of information objects or sources. Once people open a new Web page, they make an evaluative judgment in terms of how good the information is, how useful the information is, how trustworthy the information is, how accurate the information is, and so on. The reasons underlying such judgments are based on certain characteristics of information objects and sources. If people find that evaluative judgments of the information do not match the expectations of the earlier predictive judgments, they might return to a previous page or decide to start with a new page. By iterating the process, people can reach a point at which their predictive judgments and evaluative judgments match and they will proceed to use that information. ${ }^{[21]}$

Wathen and Burkell's model of credibility assessment, developed by synthesizing the literature in the field, also proposes that the assessment of credibility of online information is iterative. ${ }^{[2]}$ Upon entering a Web site on the first level of evaluation, people rate the credibility of the medium in question based on surface characteristics such as appearance, interface design, download speed, interactivity, and organization of information. Once the site passes people's credibility criteria in the initial evaluation, they then move to the next level of evaluation. Should the site fail the first evaluation, people are likely to leave the site to seek others. On the second level of evaluation, people consider the credibility of the source and the message, evaluating source expertise, competence, trustworthiness, and credentials. The message is evaluated in terms of content, relevance, currency, accuracy, and tailoring. On the third level of the evaluative process, the interaction of message presentation and content can be assessed with respect to people's cognitive states. Wathen and Burkell point out that, given the limitless number of possible interactions among contextual and intervening variables, credibility assessment becomes quite complex and difficult to predict.

Fogg's Prominence-Interpretation Theory grew out of a series of research projects conducted at the Stanford Web Credibility Research Lab. ${ }^{[23]}$ Fogg's theory describes the credibility assessment process from the point at which people notice something of interest in a Web site to the point at which they make their judgment. This theory states that two events need to occur for people to make credibility assessments on the Web: they notice element(s) in a Web site (prominence), and make judgments about what has been noticed (interpretation). If people do not notice the element, it would not have any impact on their credibility assessment of the site. Fogg's theory proposed five factors affecting prominence: involvement (motivation and ability to scrutinize Web site content), topic of the Web site, task of the user, experience of the user, and individual differences in users. Interpretation, the theory's second component, involves people's judgments about a Web site element in terms of being good or bad. Various factors relate to interpretation, including assumptions (culture, past experiences, heuristics, etc.), skills/knowledge (level of competency in the site's subject matter), and context (environment, expectations, situational norms, etc.). This process of prominence and interpretation can occur 
more than once because new aspects of the site can be continually noted and interpreted in the process of making credibility assessments on the Web.

A number of credibility researchers have observed that people do not always engage fully in the cognitive effort of making analytical judgments of content messages and sources. Rather, people often rely on mental shortcuts to judgmental rules (or heuristics), which have evolved as generalizations in their knowledge base and have thus been refined through the course of their experience. ${ }^{[24]}$ People are often unaware of the role of heuristics in influencing their judgments, which can result in their accepting a message as credible without first attributing the specific reasons for their acceptance. Cues that trigger heuristics can either be embedded in a message or internally located within people's cognition. ${ }^{[25]}$

Sundar's research team at the Media Effects Research Laboratory at Pennsylvania State University identified four broad affordances in digital media capable of cueing cognitive heuristics pertinent to making credibility assessments: modality (M), agency (A), interactivity (I), and navigability $(\mathrm{N}) .{ }^{[26]}$ The modality affordance, which often appears in the multimedia in digital devices, is the most structural (that is, tied to structure rather than to content) and apparent on an interface and accordingly triggers heuristics related to realism, novelty, and coolness, among factors. The agency affordance relates to identification of the source. Depending on who or what is perceived as a source, cognitive heuristics are triggered and in turn affect the information's perceived credibility. The interactivity affordance of digital media provides cues related to interaction, activity, choice, control, and responsiveness. Finally, the navigability affordance has dual abilities: hyperlinks on a Web site may trigger heuristics given that easily navigable sites have perceived credibility, and words on the hyperlink trigger different heuristics more closely related to the nature of the content. These four affordances are all structural features that help explain the perceived credibility of digital media beyond content characteristics. The affordances are associated with first impressions of surface-level characteristics of Web sites, which are capable of amplifying or diminishing content effects on credibility. The core idea of Sundar's model is that, while cues and heuristics do not guarantee success, they likely appeal to many individuals striving to cope with the deluge of information.

Hilligoss and Rieh's credibility framework also considers heuristics as an important component of credibility assessment. ${ }^{[27]}$ The results of an empirical study about people's credibility assessment in a variety of everyday life information-seeking contexts identified three distinct levels of credibility judgments: construct, heuristics, and interaction. The construct level involves defining the notion of credibility that influences people's judgments. The heuristics level pertains to general rules of thumb for credibility assessment, applicable to a variety of general information seeking situations. The interaction level refers to credibility judgments in which particular sources or content cues are characterized. These three levels of credibility assessment are interlinked; for instance, people's constructions of credibility influence the kind of heuristics used in selecting a Web site in which people begin a search. Credibility heuristics can influence the ways in which people pay attention to certain characteristics of information and sources. As people gain more experience with a certain source of information, credibility heuristics can be changed or extended. Should a heuristic prove consistent over time, then it becomes a construct of credibility in people's minds. Their model additionally demonstrates that context is a factor importantly influencing all three levels of credibility assessment. The context is the social, relational, and dynamic frames surrounding people's information-seeking processes, creating boundaries around the informationseeking activity or the credibility judgment itself. The context of credibility judgments can either guide the selection of resources or limit judgment applicability.

\section{CONCLUSION}

This entry intends to raise awareness about notions of information credibility and cognitive authority in the field of LIS as well as to encourage additional research work in this area. The entry defines two concepts, introduces key terms and dimensions, and discusses major theories and models proposed and tested in LIS and related fields. It also lays out the foundations of credibility and cognitive authority in historical contexts in order to illustrate the importance of understanding and enhancing these concepts as foci of research and practice.

Assessment of credibility and cognitive authority is a ubiquitous human activity, given that people constantly make decisions and selections based on the value of information in a variety of school, work, and everyday life contexts. Further, credibility and cognitive authority assessment can be seen as an ongoing and iterative process rather than a discrete evaluation event. The judgments made in assessment processes are highly dependent upon people's accumulated beliefs, existing knowledge, and prior experiences. While information objects, sources, and media often provide clues and bases for assessment, it is eventually people who make assessment with respect to their information seeking goals and motivations.

Credibility and cognitive authority have long been significant concepts for both researchers and practitioners in LIS and communication fields. Theoretical developments and applications are still emerging and evolving given changing information and communication technology environments. Previous credibility research has looked at people as information consumers while holding rather narrow views of information activities, for example, by 
focusing on seeking, reading, receiving, and watching. Today, more people are engaging in a broad range of information activities, such as creating and mediating content while actively utilizing new and diverse information technology tools and applications. Further empirical studies need to be conducted to investigate new kinds of research problems that reflect dynamic and complex information seeking and use contexts. Additional broad and multidisciplinary theoretical frameworks need to be constructed to represent a variety of human information behaviors beyond information seeking and retrieving. Creative and insightful inquiries from researchers and practitioners into credibility and cognitive authority in the LIS field would lead to efficient systems and programs that will eventually help people become more effective information users.

\section{REFERENCES}

1. Tseng, S.; Fogg, B.J. Credibility and computing technology. Commun. ACM 1999, 42 (5), 39-44.

2. Wilson, P. Second-Hand Knowledge: An Inquiry into Cognitive Authority; Greenwood Press: Westport, CT, 1983.

3. Self, C.C. Credibility. In An Integrated Approach to Communication Theory and Research, Salwen, M.B., Stacks, D.W., Eds.; Lawrence Erlbaum Associates, Inc.: Mahwah, NJ, 1996; 421-441.

4. Hovland, C.I.; Janis, I.L.; Kelley, H.H. Communication and Persuasion; Yale University Press: New Haven, CT, 1953.

5. Fogg, B.J. Persuasive Technology: Using Computers to Change What We Think and Do; Morgan Kaufmann: San Francisco, CA, 2003.

6. Flanagin, A.J.; Metzger, M.J. Digital media and youth: Unparalleled opportunity and unprecedented responsibility. In Digital Media, Youth, and Credibility; Metzger, M.J., Flanagin, A.J., Eds.; MIT Press: Cambridge, MA, 2008; 5-28.

7. Saracevic, T. Relevance: A review of the literature and a framework for thinking on the notion in information science. Part II. Adv. Librarianship 2006, 30, 3-71.

8. Vickery, B.C. Subject analysis for information retrieval. In Proceedings of the International Conference on Scientific Information; National Academy of Sciences: Washington, DC, 1959; Vol. 2, 855-865.

9. Cooper, W.S. On selecting a measure of retrieval effectiveness. Part 1: The "subjective" philosophy of evaluation. J. Am. Soc. Inform. Sci. 1973, 24 (2), 87-100.

10. Maglaughlin, K.L.; Sonnenwald, D.H. User perspectives on relevance criteria: A comparison among relevant, partially relevant, and not-relevant judgments. J. Am. Soc. Inform. Sci. Technol. 2002, 53 (5), 327-342.

11. Rieh, S.Y. Judgment of information quality and cognitive authority in the Web. J. Am. Soc. Inform. Sci. Technol. 2002, 53 (2), 145-161.
12. Rieh, S.Y.; Hilligoss, B. College students' credibility judgments in the information seeking process. In Digital Media, Youth, and Credibility; Metzger, M.J., Flanagin, A. J., Eds.; MIT Press: Cambridge, MA, 2008; 49-72.

13. O'Keefe, D.J. Persuasion: Theory and Research; Sage Publications: Newbury Park, CA, 1990.

14. Metzger, M.J.; Flanagin, A.J.; Eyal, K.; Lemus, D.R.; McCann, R.M. Credibility for the 21st century: Integrating perspectives on source, message, and media credibility in the contemporary media environment. In Communication Yearbook, Kalbfleisch, P.J., Ed.; Lawrence Erlbaum Associates, Inc.: Mahwah, NJ, 2003; Vol. 27, 293-335.

15. Wilson, E.J.; Sherrell, D.L. Source effects in communication and persuasion research: A meta-analysis of effect size. J. Acad. Market. Sci. 1993, 21 (2), 101-112.

16. Petty, R.E.; Cacioppo, J.T. The Elaboration Likelihood Model of persuasion. In Advances in Experimental Social Psychology, Berkowitz, L., Ed.; Academic Press: New York, 1986; Vol. 19, 123-205.

17. Roper, B. Public Attitudes Toward Television and Other Media in a Time of Change; Television Information Office: New York, 1985.

18. Danielson, D.R. Web credibility. In Encyclopedia of Human-Computer Interaction; Ghaoui, C., Ed.; Idea Group, Inc.: Hershey, PA, 2005; 713-721.

19. De George, R.T. The Nature and Limits of Authority; University Press of Kansas: Lawrence, KS, 1985.

20. Taylor, R.S. Question negotiation and information seeking in libraries. Coll. Res. Libr. 1968, 29, 178-194.

21. Rieh, S.Y.; Belkin, N.J. Interaction on the Web: Scholars' judgment of information quality and cognitive authority. In Proceedings of the 63rd Annual Meeting of the American Society for Information Science; Chicago, IL, November 13-16, 2000; Kraft, D.H., Ed.; Information Today: Medford, NJ, 2000; Vol. 37, 25-38.

22. Wathen, C.N.; Burkell, J. Believe it or not: Factors influencing credibility on the Web. J. Am. Soc. Inform. Sci. Technol. 2002, 53 (2), 134-144.

23. Fogg, B.J. Prominence-interpretation theory: Explaining how people assess credibility online. In Proceedings of the SIGCHI Conference on Human Factors in Computing Systems, Fort Lauderdale, FL, April 5-10, 2003; Cockton, G., Korhonen, P., Eds.; ACM: New York, 2003; 722-723.

24. Chaiken, S. Heuristic versus systematic information processing and the use of source versus message cues in persuasion. J. Pers. Soc. Psychol. 1980, 39 (5), 752-766.

25. Chen, S.; Chaiken, S. The heuristic-systematic model in its broader context. In Dual-Process Theories in Social Psychology; Chaiken, S., Trope, Y., Eds.; Guilford Press: New York, 1999; 73-96.

26. Sundar, S.S. The MAIN Model: A heuristic approach to understanding technology effects on credibility. In Digital Media, Youth, and Credibility; Metzger, M.J., Flanagin, A.J., Eds.; MIT Press: Cambridge, MA, 2008; 73-100.

27. Hilligoss, B.; Rieh, S.Y. Developing a unifying framework of credibility assessment: Concept, heuristics, and interaction in context. Inform. Process. Manag. 2008, 44 (4), 1467-1484. 


\section{BIBLIOGRAPHY}

1. Fogg, B.J. Persuasive technology: Using Computers to Change What We Think and Do; Morgan Kaufmann: San Francisco, CA, 2003.

2. Metzger, M.J.; Flanagin, A.J., Eds. Digital Media, Youth, and Credibility; MIT Press: Cambridge, MA, 2008.

3. Metzger, M.J.; Flanagin, A.J.; Eyal, K.; Lemus, D.R.; McCann, R.M. Credibility for the 21st century: Integrating perspectives on source, message, and media credibility in the contemporary media environment. In Communication Yearbook; Kalbfleisch, P.J., Ed.; Lawrence Erlbaum Associates, Inc.: Mahwah, NJ, 2003; Vol. 27, 293-335.

4. Rieh, S.Y.; Danielson, D.R. Credibility: A multidisciplinary framework. In Annual Review of Information Science and Technology, Cronin, B., Ed.; Information Today: Medford, NJ, 2007; Vol. 41, 307-364.

5. Wilson, P. Second-Hand Knowledge: An Inquiry into Cognitive Authority; Greenwood Press: Westport, CT, 1983. 